\title{
Mehr Krebspatienten, aber immer weniger Onkologen
}

der Versorgung aus. „In Summe sehen wir einen massiven Bedarf an Krebsspezialisten", betonte die DGHO-Vorsitzende Diana Lüftner, Berlin. Um die onkologische Versorgung dennoch sicherzustellen, brauche es neue Versorgungskonzepte: Die DGHO setzt vor allem in der Fläche auf Netzwerke. Ein großer Teil des

Die Zahl der Krebsneuerkrankungen in Deutschland wird in den nächsten Jahren um rund ein Sechstel ansteigen. Doch die Versorgung der Patienten ist gefährdet, warnen Onkologen. Denn der ärztliche Nachwuchs fehlt.

m Vergleich zu 2008 wird die Zahl der Krebsneuerkrankungen bis zum Jahr 2020 um etwa 67.000 pro Jahr zunehmen - ein Plus von $14 \%$. Das geht aus einer Studie der Universität Greifswald im Auftrag der Deutschen Gesellschaft für Hämatologie und Medizinische Onkologie (DGHO) hervor, die im Februar im Rahmen einer Pressekonferenz in Berlin vorgestellt wurde. Ursache dieses enormen Anstiegs sei die demografische Entwicklung. Die DGHO schaffe mit der Studie die Voraussetzung für eine sachliche, nicht interessengeleitete Abschätzung des stationären und ambulanten Versorgungsbedarfs in der Onkologie, erläuterte Mathias Freund, Rostock, neuer Geschäftsführender Vorsitzender der Fachgesellschaft.

Der Verband zeigte sich alarmiert: Für die Versorgung habe diese Entwicklung dramatische Konsequenzen. Es gebe einen Mehrbedarf in der Behandlung, gleichzeitig fehlten Onkologen. Die größten absoluten Anstiege soll es der Studie zufolge bei Krebsentitäten mit einem Gipfel im höheren Lebensalter geben, wie etwa bei Prostatakrebs und Brustkrebs. So würden pro Jahr 12.100 Männer mehr an Prostatakrebs erkranken und 5.500 Frauen mehr an Brustkrebs. Gab es 2008 in Deutschland etwa 1,3 Millionen Menschen mit einer Krebserkrankung, so würden es im Jahr 2020 etwa 1,5 Millionen sein, erklärte Studienleiter Wolfgang Hoffmann, Greifswald. Diese Entwicklung werde sich auch auf die Versorgung auswirken - und zwar sowohl in den Kliniken als auch in den Praxen.

\section{DGHO fordert mehr hochqualifizierte Onkologen}

Künftig müsse es deutlich mehr hochqualifizierte Onkologen geben. Zudem sei vor allem die Behandlung älterer Patienten sehr zeitintensiv - und die Zunahme der Krebsneuerkrankungen betreffe in allererster Linie die Älteren. „Der dringend benötigte ärztliche Nachwuchs fehlt jedoch“, warnte Freund. Ein Grund dafür sei, dass es zu wenig Weiterbildungsplätze gebe. Darüber hinaus schieden in den nächsten sieben Jahren $25 \%$ aller Hämatologen und Onkologen altersbedingt aus

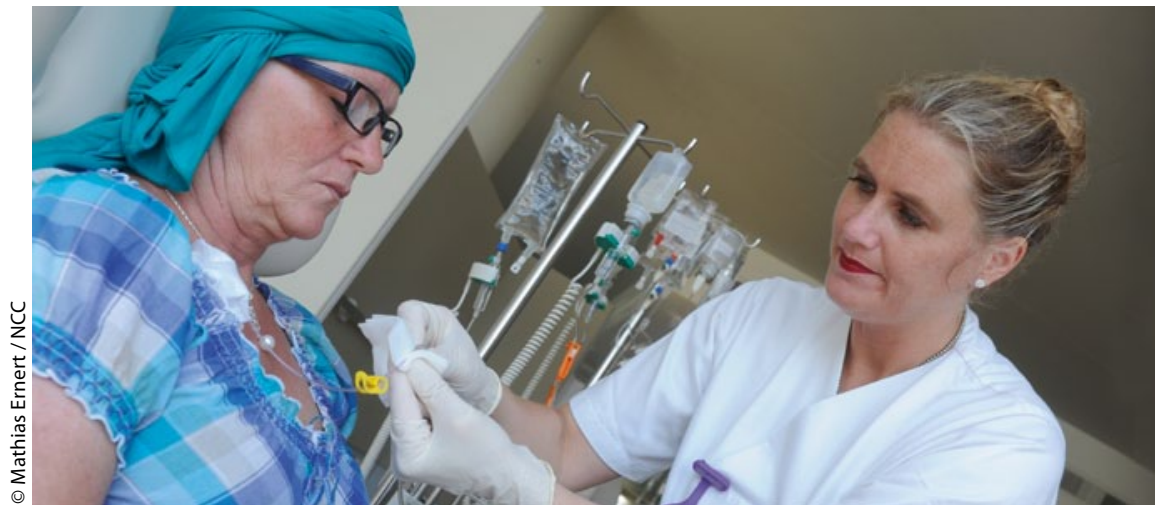

In den kommenden Jahren wird die Anzahl der Krebspatienten steigen. Damit diese Patienten adäquat versorgt werden, gilt es, frühzeitig Strategien zu entwickeln.
Mehrbedarfs dürfte im ambulanten Sektor entstehen. Leistungen würden zunehmend aus dem stationären in den ambulanten Sektor verschoben.

Aus Sicht der DGHO untermauern die Ergebnisse der Greifswalder Studie auch die wachsende Bedeutung der Medizinischen Onkologie für die Versorgung von Krebspatienten in Deutschland. „Medizinische Onkologen sind die Spezialisten für die konservative Krebstherapie. Ihnen kommt gerade bei älteren Krebspatienten eine ganz zentrale Stellung zu, da das Management der Nebenwirkungen eine solide internistische Basis erfordert", so Freund.

\section{Medizinische Onkologie stärken}

Die DGHO setzt sich deswegen dafür ein, dass das EU-weit etablierte Fach „Medical Oncology“ als „Medizinische Onkologie“ auch in Deutschland einen höheren Stellenwert bekommt. Durch die Einrichtung eigenständiger Lehrstühle für Medizinische Onkologie könnten die Sichtbarkeit des Fachs für den akademischen Nachwuchs verbessert und mehr junge Ärzte für eine klinische und wissenschaftliche Tätigkeit in der Onkologie gewonnen werden. Um die Bedeutung des Fachs auch nach außen zu signalisieren, hat die DGHO eine Präzisierung ihres Namens in „Deutsche Gesellschaft für Hämatologie und Medizinische Onkologie e. V.” vorgenommen. „Wir sehen einen engen Zusammenhang zwischen der Entwicklung in Deutschland und in Europa", betont Freund. Auf europäischer Ebene wurde erst kürzlich das Fach „Medical Oncology“ als medizinische Spezialität anerkannt. Allerdings spielen auch noch andere Berufsgruppen bei der Versorgung der Krebspatienten eine Rolle, betonten die Vertreter der DGHO. Das solle nicht vergessen werden. Neben anderen Facharztgruppen seien insbesondere auch Pflegekräfte, Physiotherapeuten und Psychotherapeuten in die Versorgung eingebunden.

Sunna Gieseke 